\title{
Research about the Influencing Factors of Inter-organizational Knowledge Transferring from Enterprise Alliance perspective
}

\author{
YueFeng Zhou ${ }^{1,}$ a , XueQing Jing ${ }^{1, b}$ \\ ${ }^{1}$ College of Economics and Management, Dalian University, Dalian, Liaoning, 116622, China \\ aemail: zhouyf66@263.net, bemail: ajingxueqing@163.com
}

\begin{abstract}
Keywords: Enterprise alliance; Nodal capability; Inter-organizational Knowledge Transferring; Environment of alliance
\end{abstract}

\begin{abstract}
In order to study the influencing factors of inter-organizational knowledge transferring within enterprise alliance, it analyzes the factors from the capability of node and the environment of alliance, builds up a model where the absorptive capacity of the recipient and the transmit capability of the dispatcher affect the average level and the transferred rate of allied knowledge, and simulates the model to demonstrate the effect through changing the parameter by the MATLAB system. The result of the simulation explains the absorptive capacity of the recipient and the transmit capability of the dispatcher play rather positive roles on the average level and the transferred rate of allied knowledge.
\end{abstract}

\section{Introduction}

In order to improve the competition of enterprise in the market, enterprises establish strategic alliance to provide a long-term platform for sharing and transferring of knowledge resource [1-2]. Before knowledge transferring the enterprise alliance first assess knowledge potential of each node [3-4]. If the knowledge potential different of nodes in all fields is small, the enterprises alliance cannot meet the requirement of nodes so that it dismisses in final; if the knowledge the potential difference in some field is the bigger, the enterprise can produce strong willingness to learn external knowledge. And through the combination, the combination and integration phase of knowledge, the knowledge is used to enhance the enterprise competitive advantage in the market for itself. But the effective knowledge transferring process between inter-organizations within enterprise alliance is affected by many factors.

\section{Factor Analysis of inter-organizational Knowledge Transferring}

In the enterprise alliance, prompting some purposeful knowledge transfer between organizational factors include the node itself not only the ability but also including the union for the creation of environmental factors [5-6].

The node ability level angle mainly analyses from the knowledge recipient absorptive capacity and knowledge of the sender's ability to send these two aspects of knowledge transfer impact study on the factors [7]. Due to the knowledge base and structure of the individual nodes is different, so the knowledge absorptive capacity of the recipient's also corresponding different and the node continually absorbing knowledge to improve their own knowledge reserves, node absorptive capacity will gradually increase. When companies constantly enrich accumulation of knowledge, for knowledge absorptive capacity will also increase, reduce the cost of knowledge transfer, knowledge will eventually value scale implementation. So the paper regards the knowledge absorbing extent of the recipient as one of the key indicators measuring the impact of knowledge transferring. The knowledge sender owns right description and expression to the knowledge, so it has good communication with the recipient, the knowledge transferring capacity has also increased significantly, and ultimately it expands the value-added that knowledge transferring brings. This paper argues that the transmission capacity of the sender has closely connection with knowledge 
transferring within inter-organizations; when the code of knowledge is accurate, the efficiency of the knowledge transferring will advance.

The paper thinks the knowledge potential difference is considered as the sender and the receiver have knowledge stock or knowledge gap, and regards it as one of the driving forces of business alliances for the organization knowledge transferring. When knowledge potential exists asymmetry, in the low potential of the organization will seek knowledge to make up for the lack of knowledge of potential difference. This eventually led to the learning process knowledge transfer between organizations. This paper argues that the level of trust in the inter-organizational knowledge transfer process plays an essential. A high degree of trust between enterprises so that the knowledge of the sender willingness to transfer knowledge, but also allows knowledge receiver confidence in the reliability and value of the knowledge. And the trust mechanism Union will significantly reduce transaction costs between the business organization and coordination costs, and to improve the speed and flow of knowledge transfer between organizations.

\section{Numerical Model}

The paper simulates the inter-organizational knowledge transferring process within alliance based on MATLAB numerical software. The model effectively analyzes the average level and transferring rate of the enterprise alliance by changing the factors of the inter-organizational knowledge transferring.

Assume that $G=(V, E)$ is established by a number of small and medium enterprises unions, Where $V=\{1,2, \ldots, N\}$ is the league organization node, $E=\left\{e_{i j} \mid i, j \in V\right\}$ represents the alliance members directly connected nodes. When $i, j$ exist $e_{i j}=1$ or else $e_{i j}=0$, suppose node $i$ in the body of the union and its direct links to all nodes consisting of the set $G_{i}$.of course, also includes the junction point $i$. Assumptions union $t$ time for knowledge transfer, knowledge of the sender node potential is denoted by $S_{j, t}$, When $T=0$, assuming that the memory in the geopotential significant proportion of the sender node $\theta$ to $S_{0}$ in a field of knowledge union. Knowledge level of knowledge to potential low junction $H_{0}$, When $T=\mathrm{t}$, both nodes during the process of knowledge transfer, knowledge potential difference $S_{j, t}-H_{j, t}$, assume that nodes $i$ in knowledge innovation ability as the $\gamma$, Assuming knowledge of the sender sends the ability for $b\left(H_{j, t}\right)$, Assuming knowledge of the knowledge absorptive capacity for $a\left(H_{i, t}\right)$, Assumes that the league average degree of trust between organizations in the body as $w$, where $0 \leq w \leq 1$. The initial value is set, $T=150$ is termination condition for the operator, in $T=0$ moment of node of high level of knowledge is $S_{0}=10$. The remainder of the initial level of $H_{0}=10$.

Based on strategic alliance knowledge flow characteristics, finally established the alliance of two indicators to reflect the whole process of knowledge transfer: unions overall average level of knowledge and knowledge transfer speed as a whole.1) the average of the overall knowledge of the union. Union body junction point in $T=t+1$ knowledge can be expressed as Eq.1

$$
H_{i, t+1}=H_{i, t}+\Delta H_{i, t+1}
$$

This time in $t \rightarrow t+1$, the amount of increase in knowledge can be expressed as Eq.2

$$
\Delta H_{i, t+1}=\sum_{i, j \in G_{i}} a\left(H_{i, t}\right) b\left(S_{j, t}\right) \gamma\left(S_{j, t}-H_{i, t}\right) e_{i j}+\gamma H_{i, t},\left\{\begin{array}{c}
0 \leq \gamma \leq 1 \\
0 \leq b \leq 1 \\
0 \leq \gamma H_{i, t} \leq S_{t} \\
a(0)=0
\end{array}\right.
$$

So, when the time $T=\mathrm{t}$, the overall knowledge of the Union average level can be expressed as Eq.3. 


$$
\overline{H_{t}}=\frac{1}{N} \sum_{i \in G}\left[\theta N S_{i, t}+(1-\theta) N H_{i, t}\right]
$$

2) Transfer rate of the overall knowledge of the union. Union's overall knowledge transfer rate can be defined as the mean of the union all nodes per unit time knowledge transfer incremental. The union $t \rightarrow t+1$ moment of transfer rate can be expressed as Eq.4.

$$
\bar{V}_{t \rightarrow t+1}=\frac{1}{N} \sum_{i \in G} \Delta H_{i, t}
$$

Union as a whole knowledge transfer rate to reflect the Union's overall knowledge transfer efficiency, If average overall knowledge of the Union and the Union's overall knowledge transfer rate is low, the efficiency of knowledge transfer is not the best; dragged over the Union's overall knowledge transfer rate is also higher, then the organization of knowledge transfer to achieve the desired effect, the fastest in the league better knowledge transfer.

\section{The simulation results}

The model analyses the impact of knowledge transferring from the absorptive capacity of the recipient. Assume $b=0.4, \gamma=0.2, W=0.5, a$ is different value respectively. Union overall knowledge of the average transfer rate of change at different times $T$, Fig. 1 presents different absorption capacity of the Union average level of knowledge. Union as a whole the average level of knowledge is the rapid increase in the first period and eventually reaches a steady state, and the absorptive capacity of the node is higher, the Union's overall knowledge of the higher average. With the enhancement of the absorption capacity of the node, the node is reached at the same time, knowledge equilibrium time is shorter. Fig.2 presents different absorption capacity of the Union knowledge transfer speed, From Fig. 2 can get alliance knowledge transfer speed in the initial growing over a period of time, then gradually decline eventually in a relatively stable state; With the continuous improvement of absorption capacity and speed of enterprise integration and external knowledge also gradually increase, more nodes can quickly reach equilibrium.

The model analyses the impact of knowledge transferring from the absorptive capacity of the sender. The initial value unchanged, once again assumes $a=0.1, \gamma=0.2, W=0.5, b$ is different value respectively. Analysis of alliance as a whole the average level of knowledge and transfer rate change in different time $T$.Fig. 3 presents the ability to send the average level of knowledge of the Union, when the knowledge of the sender is the more accurate description of the knowledge, the ability to transfer knowledge, the stronger, the quality and quantity of knowledge transfer of knowledge of the sender is higher, the average level of the Union's overall knowledge will be higher. Fig.4 presents the ability to send the alliance knowledge transfer speed, From figure 4 can conclude alliance knowledge transfer rate are as time is in the initial period gradually raise and then lower the final stable state; and alliance knowledge of the sender to send capacity is higher, the alliance knowledge transfer rate is higher, the speed of knowledge transfer and knowledge of the sender to send capabilities are positively related.

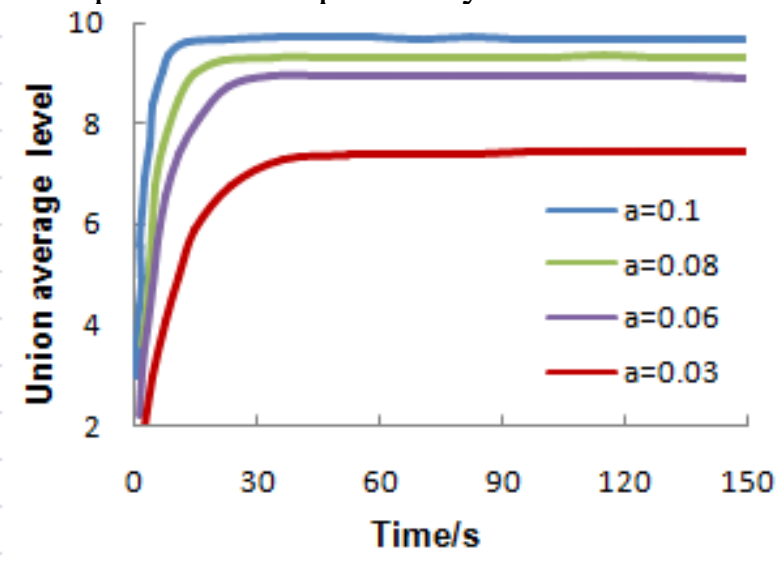

Fig.1. Absorption-union average level curve

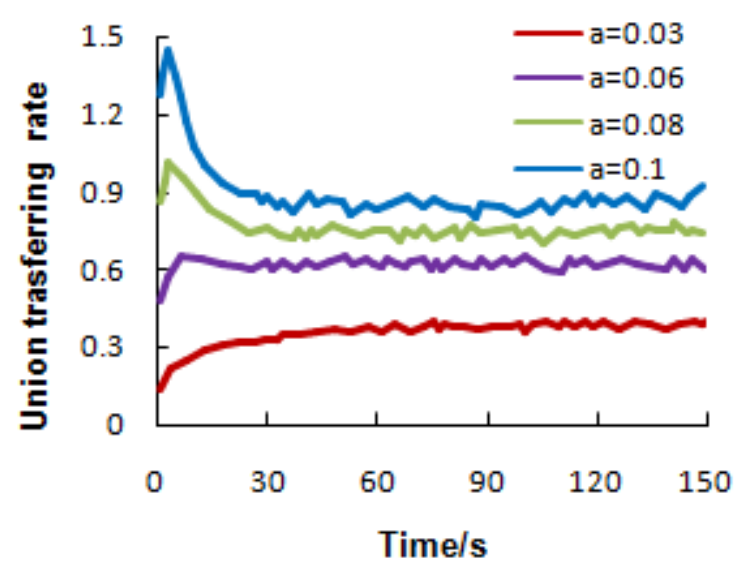

Fig.2. Absorption-union transferring rate curve 


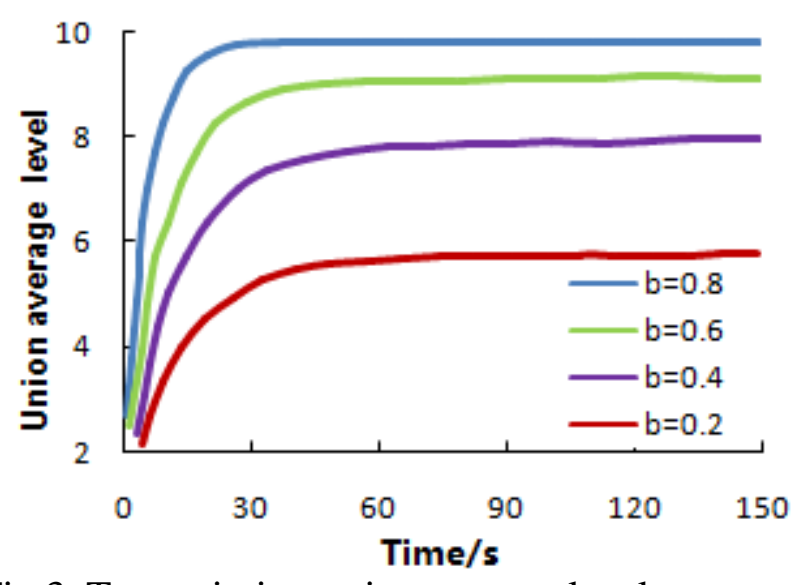

Fig.3. Transmission-union average level curve

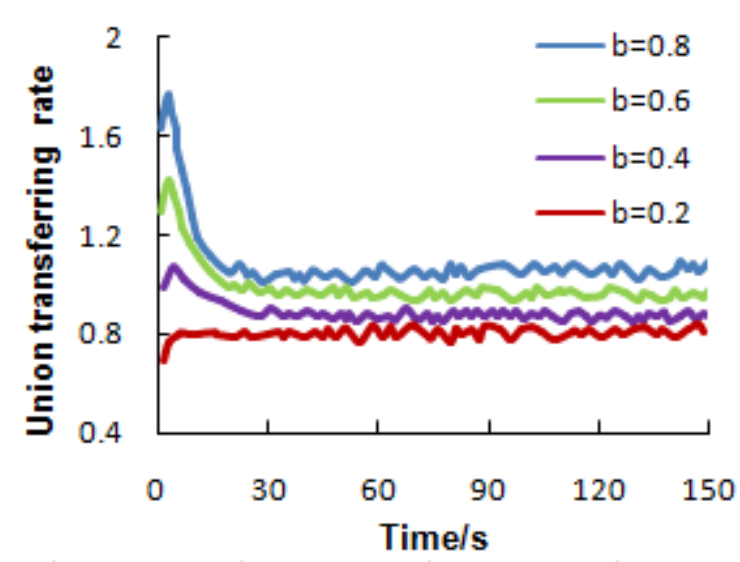

Fig.4. Transmission-union transferring rate curve

\section{Conclusion}

Inside the enterprise alliance, the influence factors of knowledge transfer in the process of inter-organizational learning is very complicated. This article from the node capacity level and environment level to analyze its impact on knowledge transfer, and to establish indicators of knowledge transfer as alliance knowledge transfer rate and average level of whole, thus constructs the influence model of inter-organizational knowledge transfer. At the same time, this article is based on the MATLAB software to change the parameters of the variables in the model to show: the node hierarchy knowledge receiver accept ability and knowledge of the sender to send capacity of knowledge transfer plays a positive role; union level knowledge of potential difference and trust mechanism of knowledge transfer between organizations also play a key role. So, to make organizational knowledge can be transferred effectively, knowledge receiver, sender and alliance environment should take is conducive to knowledge transfer in the process of inter-organizational learning strategies.

\section{References}

[1] Y.L. Wang, Y. Li, Knowledge transfer characteristics of the study. Systems Engineering-theory \& Practice, Volume22 (10), PP8-10, 2002.

[2] D.C. Wang, Y.L. Wang, Model based on absorption capacity and knowledge transparency Knowledge Alliances. Operations Research and Management Science, Volume 15(6), pp.115-120, 2006.

[3] Cowan R, Nicolas J. Network structure and the diffusion of knowledge [J]. Journal of Economic Dynamics \& Control, 2004, 28: 1557-1575.

[4] Rajesh Rajaguru, Margaret Jekanyika Matanda. Effects of inter-organizational compatibility on supply chain capabilities: Industrial Marketing Management, pp.26-39, 2012.

[5] F.C. Tang, Y. M. X, Knowledge Transfer and dynamic behavior patterns of network organization. Systems Engineering-theory \& Practice, Volume9, pp.83-89, 2006.

[6] Li wei an, Organization complexity: motivation, control and utilization Organization complexity: motivation, control and utilization. Tianjin Social Sciences, Volume5:59 - 63, 2005

[7] X.H. Lai, W.P. Wang, Dissemination of knowledge in the knowledge-based organization of cellular automata-based process simulation study, Chinese Journal of Management. Volume 3(5):514 - 518, 2006, 